\title{
Compensation of Printer MTFs
}

\author{
Nicolas Bonnier ${ }^{a, b}$, Albrecht J. Lindner ${ }^{a, b, c}$, Christophe Leynadier ${ }^{b}$ and Francis Schmitt $^{a}$ \\ ${ }^{a}$ Institut TELECOM, TELECOM ParisTech, CNRS UMR 5141 LTCI (France) \\ ${ }^{b}$ Océ Print Logic Technologies, Créteil (France) \\ ${ }^{c}$ Ecole Polytechnique Fédérale de Lausanne, Computer Vision Laboratory (Switzerland)
}

\begin{abstract}
Preliminary experiments have shown that the quality of printed images depends on the capacity of the printing system to accurately reproduce details. ${ }^{1}$ We propose to improve the quality of printed images by compensating for the Modulation Transfer Function (MTF) of the printing system. The MTF of the printing system is measured using the method proposed by Jang and Allebach, ${ }^{2}$ in which test pages consisting of series of patches with different 1D sinusoidal modulations (modified to improve the accuracy of the results ${ }^{3}$ ) are printed, scanned and analyzed. Then the MTF is adaptively compensated in the Fourier domain, depending both on frequency and local mean values. Results of a category judgment experiment show significant improvement as the printed MTF compensated images obtain the best scores.
\end{abstract}

Keywords: MTF, printer, measure, compensation, quality, deconvolution, psychophysical experiment

\section{INTRODUCTION}

In the quest for an optimal reproduction of color images, preservation of the spatial details in images has emerged as a very important issue for perceptual quality. ${ }^{4,5}$ Thus, with the growing demand for high-quality reproduction of photographic images using inkjet printers, the interest in qualifying and quantifying the behavior of printing systems has become more and more important.

To achieve high-quality reproduction, output devices should be able to reproduce adequately all the colors and the details (high-frequency content) in the processed images. During preliminary experiments ${ }^{1}$ to visually assess the quality of printing systems, we have compared images reproduced both on an Eizo Color Edge 221 LCD monitor and with a printing system composed of an Océ inkjet printer and standard Océ uncoated paper. We have observed that images present significantly better details when displayed on the monitor than when printed on standard uncoated paper. It appears that the ability of the two systems (the monitor versus the printing system) to reproduce details are different, leading to strong differences in the perception of the spatial content in the resulting images. The ability of a system to reproduce details is captured in its Modulation Transfer Function (MTF), also called Spatial Frequency Response (SFR).

In this paper, we propose to compensate for the MTF of printing systems to enhance the quality of the output images. In Section 2 we discuss the specificities of the MTF of printing systems, then we introduce the method chosen to characterize our printing system and present the resulting measurements. In Section 3 we consider the use of existing filters to compensate for the MTF of a printing system. In Section 4 we present a new locally adaptive method to compensate for the printer MTF. Then in Section 6 we explore the concept of over-compensating the MTF. The compensation algorithm is tested (Section 7) in a psychophysical experiment with photographic images in order to test its impact on the visual appearance of images. The results of this category judgment experiment are presented and commented. The newly developed algorithm shows very good results: the printer MTF is well compensated and no visual artifacts are introduced in the images.

\section{CHARACTERIZING THE PRINTER MODULATION TRANSFER FUNCTION}

The MTF of a given system shows how much the system attenuates an input modulation signal. When considering a 1 dimensional (1D) spatial modulation, the MTF is defined as the ratio of the output amplitude $A_{\text {out }}(f)$ to the input amplitude $A_{\text {in }}(f)$ :

$$
M T F(f)=\frac{A_{\text {out }}(f)}{A_{\text {in }}(f)}
$$


where $f$ is the spatial frequency component of the 1D signal. An ideal imaging device would have an MTF equal to 1 for every frequency and orientation. Usually a printing system has low-pass characteristics: mostly only high frequencies are attenuated and low frequencies are preserved.

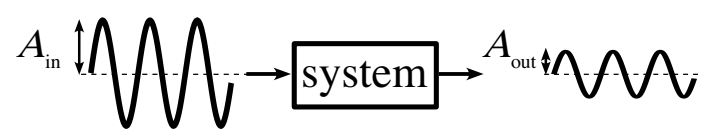

Figure 1. Attenuation of a $1 \mathrm{D}$ input sinusoidal signal with amplitude $A_{\text {in }}$ by a system resulting in a reduced output amplitude $A_{\text {out }}$.

In our preliminary experiments ${ }^{1}$ the MTF of the printing system was much lower than the MTF of the monitor. The technologies are indeed very different and the lower MTF of the printing system is mainly due to the halftoning algorithm and to the spreading of the ink on the paper.

\subsection{Existing Characterization Methods}

Characterizing the MTF of a printing system is not a trivial task: unlike digital capture devices, inkjet and laser printers are highly non-linear and therefore not easy to model. The MTF depends on different parameters related to the printing system (halftoning, addressability, dot size, quality of the medium) and on the characteristics of the input image (bias, color, spatial frequency, orientation). This is also related to the Nyquest-Shannon sampling theorem. ${ }^{6}$ In order to fully characterize or describe a printing system, the MTF has to be measured for different biases, colors, frequencies or orientations. For a modulation signal at a given set of bias, color and orientations, the results can be plotted in a graph where the MTF is plotted versus the frequency.
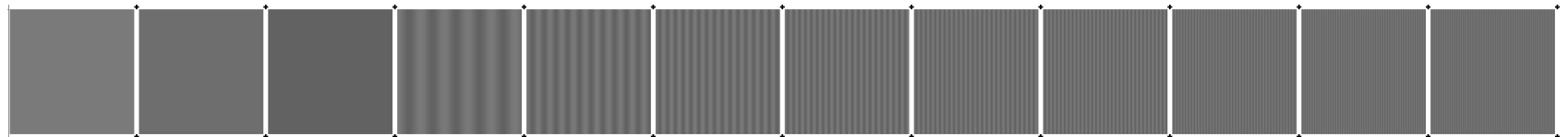

Figure 2. Example of one row of Jang and Allebach's test image. ${ }^{2}$

Characterization of the MTF has been popular in photography and there are a few methods to measure the MTF for both silver-halide and digital image capture devices, in which sinusoidal and slanted-edge targets are used. These characterization methods have been standardized by the International Organization for Standardization (ISO).$^{7,8}$ While much effort has been spent in the characterization of the color characteristic curves of printing systems, until recently little research ${ }^{2,9-11}$ has been published regarding the characterization of the MTF of printing systems. Arney et al. ${ }^{9}$ proposed a method to measure the MTF of the physical printing process without the software pre-processing, by dividing the spectrum of the printed image by the spectrum of the halftoned image. Jang and Allebach ${ }^{2}$ proposed a procedure for characterizing the MTF of a complete digital printing system, i.e. the software, the hardware and the medium in which test pages consisting of series of patches with different 1-D sinusoidal modulation are printed, scanned and analyzed (see Figures 2 and 3 ). Later Hasegawa et al. ${ }^{10}$ proposed an objective metric to evaluate the quality of the resolution of a black and white laser printer. Similarly as in Jang and Allebach, black and white stripes, lines and dot patterns are printed, scanned and analyzed, yet the analyses in the two methods are very different. The analysis is done by comparing black and white surfaces in the input image versus on the printout, thus it is mainly a measure of the dot gain. Recently, the slanted-edge method used to characterize scanners and cameras ${ }^{8}$ has been adapted by Bang et al. ${ }^{11}$ to measure the MTF of a printer: an image containing a slanted black square on white background is sent to the printer, the printout is scanned and analyzed.

After a preliminary study, ${ }^{3,12}$ we have selected the characterization method proposed by Jang and Allebach ${ }^{2}$ as it appears to be the most robust, and proposed some modifications of the test chart to further enhance it. ${ }^{12}$ 


\subsection{Jang and Allebach's Characterization}

The method proposed by Jang and Allebach ${ }^{2}$ consists of printing patches with sinusoidal patterns (Figure 2), scanning them with a high resolution scanner and comparing their amplitudes with the values of constant tone patches (Figure 3). The value of these constant tone patches correspond to the maximum (max), the mean (bias) and the minimum (min) of the sinusoidal patches in the same row. One row of the test image (as illustrated in

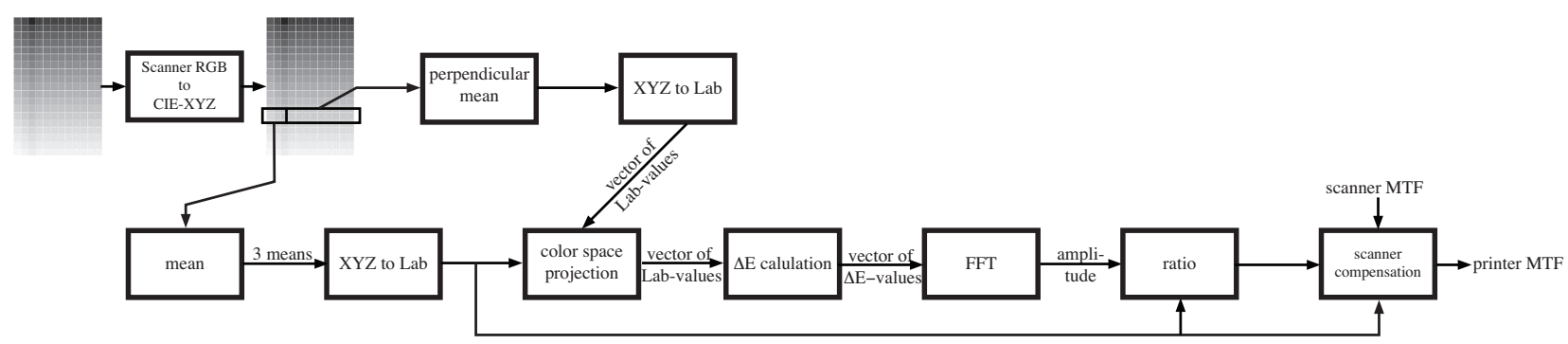

Figure 3. Flowchart of Jang and Allebach's characterization. ${ }^{2}$

Figure 2) consists of these three constant tone patches followed by nine sinusoidal patches oscillating between the min and max value with frequencies set to 10, 20, 30, 40, 50, 60, 80, 100, 150 cycles per inch, respectively. To measure the MTF with different biases, the test image consists of 19 rows. In the original test chart, ${ }^{2}$ the 19 bias values $b \in \mathcal{B}=\left\{b_{1}, b_{2}, \ldots, b_{19}\right\}$ are equidistantly distributed on $L^{*}$, the lightness scale of the CIELAB ${ }^{13}$ color space. We have proposed ${ }^{3}$ to slightly modify the test chart to improve the reliability of the results: the bias values are equidistantly distributed in CIE XYZ, ${ }^{13}$ a colorimetric space better suited as it is more related to the physics of the measurement on the printing system. Furthermore, to lessen the influence of noise in the measurement, the amplitude of modulation of the sine waves is increased to $\Delta Y=20$ (except for the extreme bias values to avoid clipping of the signal to either the paper white or the maximum black of the ink).

The characterization of a printing system is depicted in Figure 3 and can shortly be described as follows. We first scan the printout, then each row with constant mean gray level (bias) is processed separately.

1. Within one row the three constant tone patches are processed first. For each patch, its mean is calculated in the CIE XYZ space and converted into CIELAB values.

2. For each of the nine sinusoidal patches from the same row, the modulation signal is extracted by averaging the measured tristimulus values perpendicularly to the direction of modulation. The averaged values are converted to CIELAB values and projected on the line which connects the lower and upper mean values corresponding to the constant min and max tone patches, respectively.

3. Then, for all the points projected on the line the $\Delta E_{a b}^{*}$ distance to the lower mean value of the constant min patch is calculated. The result is a vector of $\Delta E_{a b}^{*}$ scalar values which is Fourier transformed. The amplitude of the main frequency of the patch is then extracted.

4. The amplitude is compared with the $\Delta E_{a b}^{*}$ distance between the constant min and max tone patches, it is usually smaller. Since the scanner is not compensated at this point, the ratio of the amplitude over the distance $\Delta E_{a b}^{*}$ is not yet the printer MTF. It is the MTF of the system composed by both the printing system and the scanner.

5. For the scanner compensation we use the scanner MTF which has been separately measured with specific engraved patterns on a physical chart. ${ }^{8}$ We then estimate how much the scanner attenuates a signal which oscillates between the min and max constant tone patches. This scanner ratio should be between 1 and the ratio calculated in the previous step. Dividing the first calculated combined printer and scanner ratio by the above scanner ratio provides the estimated compensated printer MTF. 


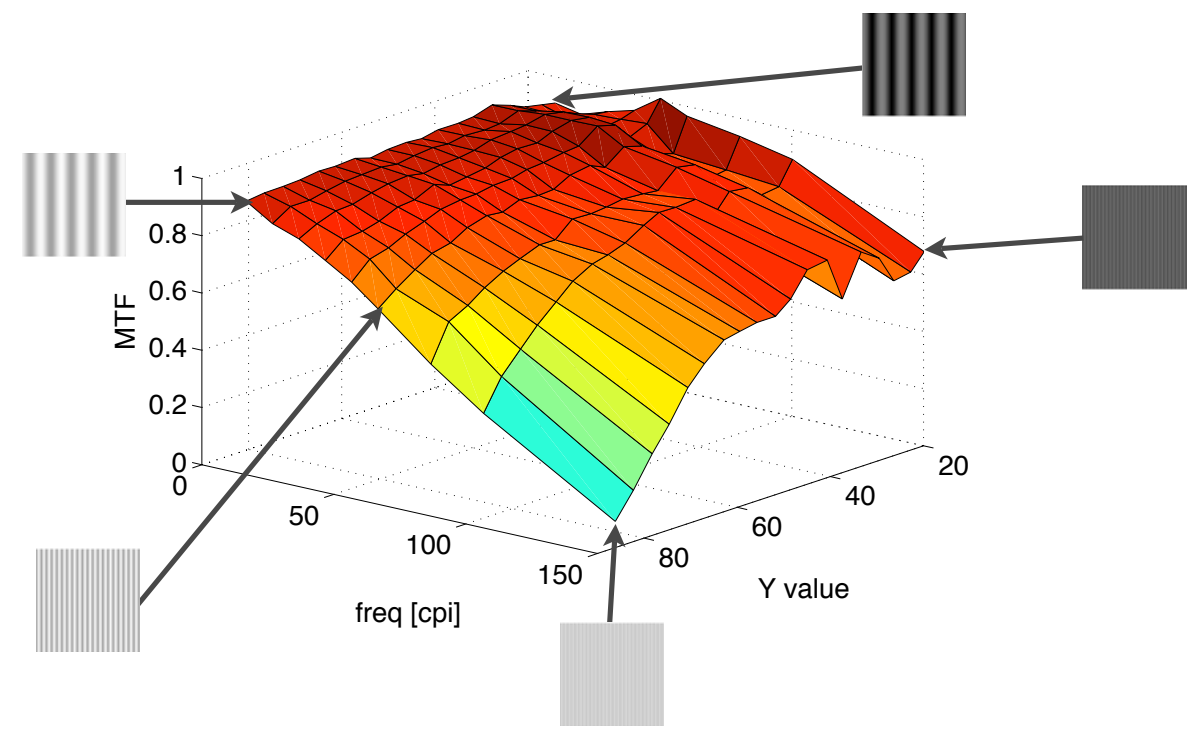

Figure 4. Measured horizontal MTF of the Océ Colorwave 600 inkjet printer on Océ uncoated paper, obtained with the modified Jang and Allebach ${ }^{2}$ test image (19 rows of increasing gray values (bias) x 9 columns of sine patterns increasing in frequency).

\subsection{Experimental MTF Measure}

In our study we have used the inkjet printer Océ Colorwave 600 with standard Océ uncoated paper and an Epson scanner Expression 10000XL. Figure 4 shows the MTF characterization obtained with the modified Jang and Allebach test chart, aligned in the horizontal direction. The MTF decreases as the frequency of the sine wave increases, as expected, but the MTF depends also on its bias or mean value: the MTF values are higher for medium gray levels than for high gray levels (high row indices) and slightly higher for low gray levels (low row indices). For this printer, we find that the MTF values for the horizontal and vertical directions are very similar. Therefore, we restrict the remaining of this study to a case where the MTF doesn't depend on the orientation. Solutions to take the orientation into account will be discussed later in Section 5 .

\section{COMPENSATING FOR THE PRINTER MTF}

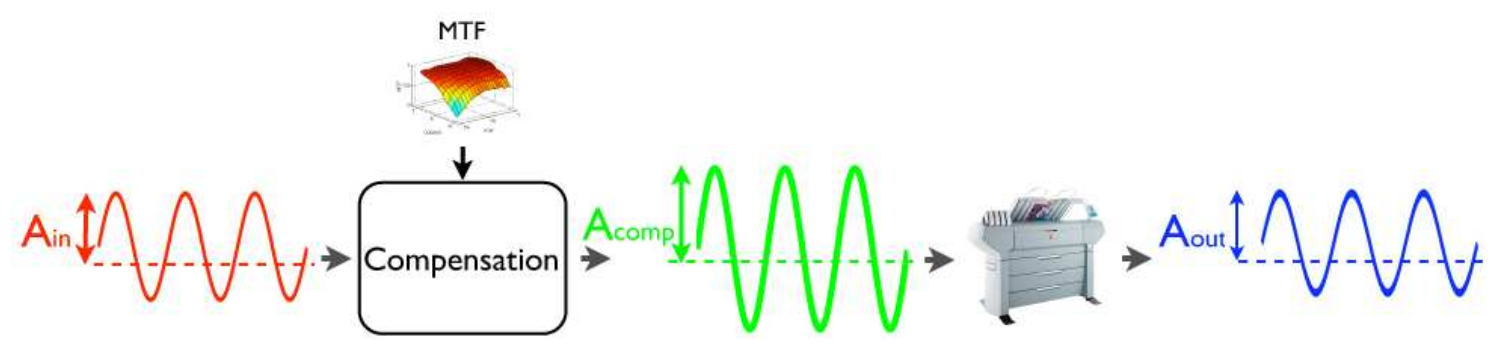

Figure 5. Principle of the MTF compensation: the Amplitude $A_{i n}$ is compensated (pre-amplified) by Compensation (division by the $M T F$ in the Fourier domain) before printing, resulting in a printed output amplitude $A_{\text {out }}$ close to the input $A_{\text {in }}$.

The initial aim of this study is to be able to compensate for the printer MTF by pre-processing the image sent to the printer as in Figure 5. The compensation of an MTF is already known from other fields as the restoration of blurred images. ${ }^{14}$ For example consider a picture of a real scene captured with a digital camera: as cameras have a low-pass characteristic, the captured image is not sharp. The MTF of the camera can be compensated to 
obtain a sharper image, closer to the real scene. While camera compensation occurs in post-processing, printer compensation must occur in pre-processing. Nevertheless it is based on the same principles, since both processes aim to amplify the frequencies by a factor given by the system MTF.

Another critical difference is that printing systems are non-linear. Therefore, an optimal compensation of the MTF should depend on different parameters related to the characteristics of the input images such as the bias, color, spatial frequency or orientation.

\subsection{Deconvolution}

Under the assumption of a linear system, the degradation can be described by a multiplication in the Fourier domain. Assuming an input image $\mathbf{I}_{i n}$, its Fourier transform being $F\left(\mathbf{I}_{i n}\right)$ and the system modulation transfer function $M T F$, the Fourier transform of the degraded image at the output is $F\left(\mathbf{I}_{\text {out }}\right)=M T F \times F\left(\mathbf{I}_{\text {in }}\right)$. In the case where $M T F$ is known at each frequency, the compensation is a simple division in the Fourier domain and the compensated input image $F\left(\mathbf{I}_{\text {comp }}\right)$ is then obtained by the inverse Fourier transform $F^{-1}$ as follows:

$$
\mathbf{I}_{c o m p}=F^{-1}\left(\frac{F\left(\mathbf{I}_{i n}\right)}{M T F}\right) .
$$

This is also known as deblurring or as a deconvolution since it is the inverse of a convolution in the spatial domain. Unfortunately this ideal case might be perturbed by noise. The system MTF has to be estimated by a measurement which itself is affected by noise. This is mostly problematic for frequencies where the system attenuation is strong, leading to low values of MTF and to numerical instabilities in the deconvolution. Furthermore, the image $\mathbf{I}_{i n}$ might also contain noise. Different filters have been proposed to take the noise into account.

\subsection{Wiener Filter}

The Wiener filter named after his author ${ }^{15}$ is a modification of the division in Equation 2 taking the noise into account. It is done by dividing through the sum of the MTF and a noise-to-signal ratio:

$$
\mathbf{I}_{c o m p}=F^{-1}\left(\frac{F\left(\mathbf{I}_{i n}\right)}{M T F} \times \frac{M T F \times M T F^{*}}{M T F \times M T F^{*}+N S R}\right),
$$

where $\mathbf{I}_{i n}$ is the input image, $\mathbf{I}_{c o m p}$ is the compensated image, MTF is the MTF of the system and $M T F^{*}$ its complex conjugate, $N S R$ is the noise-to-signal ratio of the image $\mathbf{I}_{i n}$. When the noise tends to zero, the Wiener filter reduces to Equation 2. But the more noise there is in the image, the less is the filter impact. The estimation of the noise-to-signal ratio $N S R$ might be problematic, as a printer can get an input image from any source (camera, scanner, software, ...) and every device has its own noise characteristics.

There is a variety of other possibilities to compensate a convolution kernel using different techniques (see Chan and Shen ${ }^{16}$ for an overview). One issue with all these advanced methods is that they tend to use more calculation time and storage than the simple division from Equation 2.

\subsection{Unsharp Masking}

Alternatively, unsharp masking, ${ }^{17}$ based on the subtraction of a smoothed image from the input image, can be used to increase contrast of existing edges:

$$
\mathbf{I}_{c o m p}=\mathbf{I}_{i n}+\alpha \times\left(\mathbf{I}_{i n}-f_{b l u r}\left(\mathbf{I}_{i n}\right)\right),
$$

where $f_{\text {blur }}$ is a blurring low-pass filter such as a Gaussian filter, and $\alpha$ a constant coefficient setting the amount of unsharp masking. This linear filter is frequently used in the digital photography community, usually to regain sharpness attenuated in the scanning process, or sharpen reproduction of images on prints. Limitations of such filter include the need to set a filter size in $f_{\text {blur }}$ that limits the gain of sharpness to a fixed range of frequencies. An unapropriate setting of the size might lead to halos in resulting images. Furthermore, $\alpha$ is a constant coefficient setting the amount of boosting uniformly across the fixed frequency range, when frequency-dependent coefficients would allow a more effective compensation of the MTF.

In this study preliminary tests showed that the simple division gives satisfying results, mainly due to the fact that the measured MTF values are mostly greater than 0.5. Therefore we propose to use the simple division and take into account the non-linearity of the system. 


\section{LOCALLY ADAPTIVE COMPENSATION}

The MTF values vary as a function of the mean gray level: Figure 4 shows how the MTF values in the highlights significantly differ from the ones in the mid-tones and the shadows. In preliminary test, we attempted to use the mean of MTFs over the gray bias. ${ }^{18}$ Consequently, the measured compensated MTF were too low (undercompensated) for light gray patches and too high (over-compensated) for dark gray patches.

In this section we propose a compensation algorithm based on image decomposition which locally adjusts in an image the MTF compensation given the local mean for each pixel of that image. Since the Human Visual System (HVS) is more sensitive to luminance high-frequency content than chrominance high-frequency content, we limit the deconvolution to grayscale high-frequency content of the image. Furthermore, to avoid the introduction of halos $^{19}$ we first decompose the image in two bands in the CIELAB color space, the high-pass band $\mathbf{I}_{h i g h}$ and the low-pass band $\mathbf{I}_{\text {low }}$. The compensation algorithm is then applied to $\mathbf{I}_{\text {high }}$ only.

Image Decomposition The decomposition is obtained by $5 \mathrm{D}$ Bilateral Filtering $(B F)$ in the CIELAB space as proposed by Tomasi and Manduchi. ${ }^{20}$ It is a combined spatial domain and color range filtering. Let $L_{\text {low }}^{*}=$ $B F\left(L^{*}\right)$ denote the $L^{*}$ channel of the filtered image. The $L_{\text {low }}^{*}$ value of pixel $i, L_{\text {low }}^{* i}$, can be obtained as follows:

$$
L_{\text {low }}^{* i}=\sum_{j \in \mathbf{I}_{\text {in }}} w_{B F}^{j} L^{* j}, w_{B F}^{j}=\frac{d\left(\mathbf{x}^{i}, \mathbf{x}^{j}\right) r\left(\mathbf{p}^{i}, \mathbf{p}^{j}\right)}{\sum_{j \in \mathbf{I}_{\text {in }}} d\left(\mathbf{x}^{i}, \mathbf{x}^{j}\right) r\left(\mathbf{p}^{i}, \mathbf{p}^{j}\right)}
$$

where $\mathbf{I}_{i n}$ is the input image, $d\left(\mathbf{x}^{i}, \mathbf{x}^{j}\right)$ measures the geometric closeness between the locations $\mathbf{x}^{i}$ of pixel $i$ and $\mathbf{x}^{j}$ of a nearby pixel $j, r\left(\mathbf{p}^{i}, \mathbf{p}^{j}\right)$ measures the colorimetric similarity between the colors $\left(L^{* i}, a^{* i}, b^{* i}\right)$ and $\left(L^{* j}\right.$, $\left.a^{* j}, b^{* j}\right)$ of pixels $i$ and $j$.

In our implementation, $d\left(\mathbf{x}^{i}, \mathbf{x}^{j}\right)$ and $r\left(\mathbf{p}^{i}, \mathbf{p}^{j}\right)$ are Gaussian functions of the Euclidean distance between their arguments:

$$
d\left(\mathbf{x}^{i}, \mathbf{x}^{j}\right)=e^{-\frac{1}{2}\left(\frac{\left\|\mathbf{x}^{i}-\mathbf{x}^{j}\right\|}{\sigma_{d}}\right)^{2}}, r\left(\mathbf{p}^{i}, \mathbf{p}^{j}\right)=e^{-\frac{1}{2}\left(\frac{\Delta E_{a b}^{*}\left(\mathbf{p}^{i}, \mathbf{p}^{j}\right)}{\sigma_{r}}\right)^{2}},
$$

where the two scale parameters are set to $\sigma_{d}=4 \%$ of the image diagonal and $\sigma_{r}=20 \Delta E_{a b}^{*} 21$. To compute the low-pass band $\mathbf{I}_{l o w}$, only the channel $L_{i n}^{*}$ of the input image $\mathbf{I}_{i n}$ is filtered using $5 \mathrm{D}$ bilateral filtering as described above (Eq.5-6). Nevertheless, since the $5 \mathrm{D}$ bilateral filter involves $\Delta E_{a b}^{*}$ distance, the $a^{*}$ and $b^{*}$ values are taken into account in the filtering of $L_{i n}^{*}$. The high-pass band $\mathbf{I}_{h i g h}$ is then calculated by taking the difference of $\mathbf{I}_{i n}$ and the low-pass band $\mathbf{I}_{\text {low }}$ :

$$
\begin{aligned}
\mathbf{I}_{\text {low }} & =\left(L_{\text {low }}^{*}, a_{i n}^{*}, b_{i n}^{*}\right), \\
\mathbf{I}_{\text {high }} & =\mathbf{I}_{\text {in }}-\mathbf{I}_{\text {low }}=\left(L_{\text {in }}^{*}-L_{\text {low }}, 0,0\right),
\end{aligned}
$$

with $L_{\text {low }}^{*}=B F\left(L_{i n}^{*}\right), L_{\text {high }}^{*}=L_{i n}^{*}-L_{l o w}^{*}$.

Bias Dependent Compensation We propose to take into account the image local mean gray level as an estimation of the bias when compensating for the printer MTF: each pixel in $L_{h i g h}^{*}$ is compensated according to the local mean in $\mathbf{I}_{i n}$ :

$$
L_{h i g h_{\text {comp }}}^{*}=M T F_{c o m p}\left(L_{h i g h}^{*}, L_{i n}^{*}, M T F\right)
$$

where $L_{h i g h_{\text {comp }}}^{*}$ is the compensated $L^{*}$ channel and $M T F_{\text {comp }}$ the locally adaptive compensation.

Local Mean A local measure of the mean gray level $L_{i n}^{*}$ is needed to achieve the locally adaptive compensation. Here we can take full advantage of the image decomposition and use $\mathbf{I}_{l o w}$ as an estimation of the local mean. Notice that values in $\mathbf{I}_{l o w}$ are obtained by bilateral filtering. This is an advantage as a local mean computed in $\mathbf{I}_{\text {in }}$ for a local detail in $\mathbf{I}_{\text {high }}$ should only be computed by using local and similar gray pixels, as this is done in 
$\mathbf{I}_{\text {low }}$ by the bilateral filter. Since the MTF was measured using modulation and bias values based on the CIE $\mathrm{XYZ}$ color space, $\mathbf{I}_{\text {low }}$ is converted to CIE XYZ and $Y_{\text {low }}$ is taken as the local mean:

$$
L_{\text {high }}^{*}=M T F_{\text {comp }}\left(L_{\text {high }}^{*}, Y_{\text {low }}, M T F\right)
$$

$\mathbf{N}$ Deconvolutions of $L_{h i g h}^{*}$ The MTF has been measured for the bias values $b_{n}, n \in[1, N]$. Let $M T F_{n}$ denotes the MTF measured for bias $b_{n}$. $L_{h i g h}^{*}$ can be divided through each $\mathrm{MTF}_{n}(f)$ in the Fourier domain which results in $N$ compensated high-pass images:

$$
L_{\overline{h i g h}}^{*}=F^{-1}\left(\frac{F\left(L_{h i g h}^{*}\right)}{K_{M T F_{n}}}\right), n \in[1, N],
$$

where $L_{\frac{1}{h i g h_{n}}}^{*}$ is the compensated image in the image domain for bias value $b_{n}$.

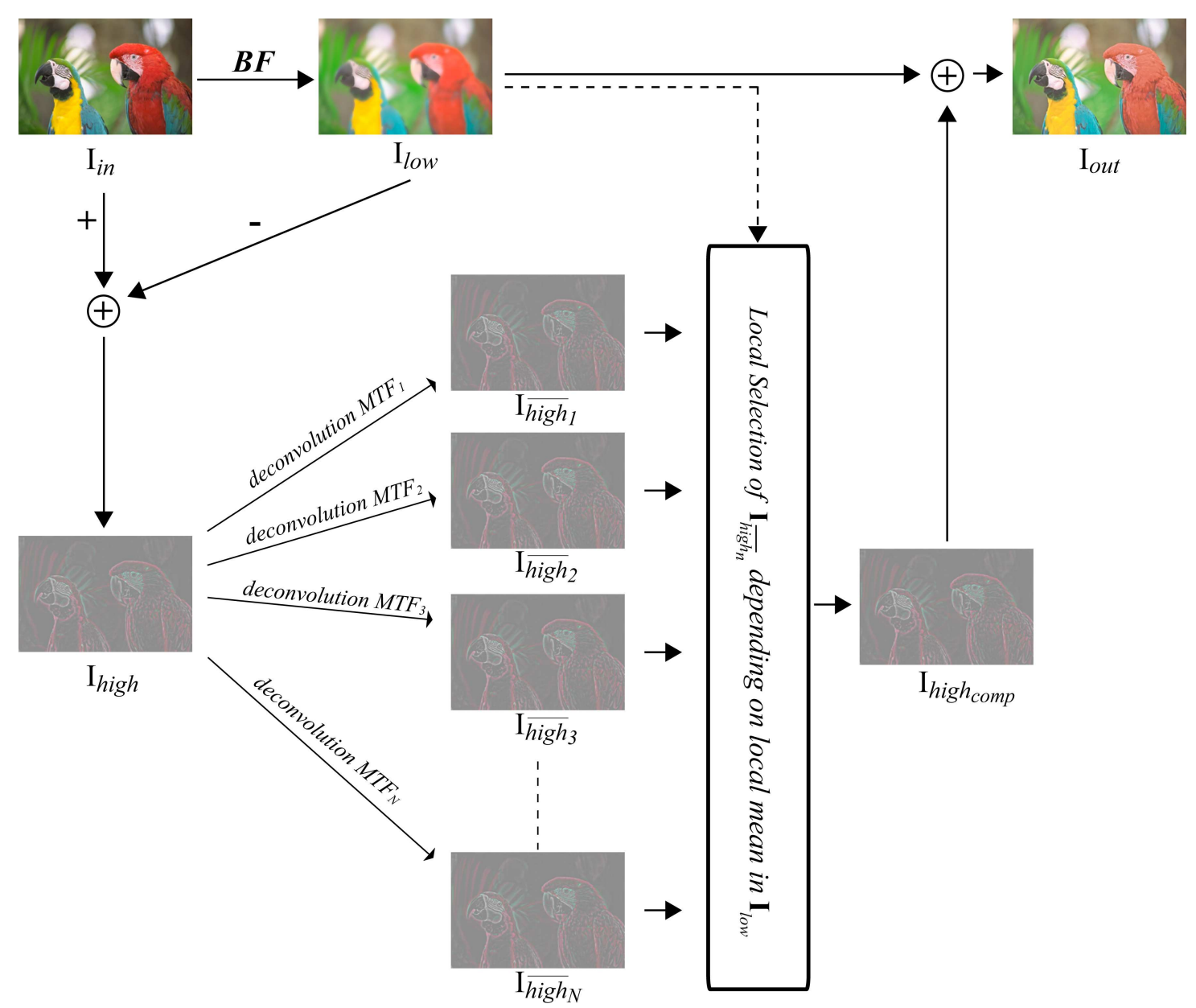

Figure 6. Principle of the locally adaptive MTF compensation: the high-pass band $\mathbf{I}_{\text {high }}$ is compensated N times for $M T F_{n}$. Then for each pixel, a locally adaptive merging is processed according to the local mean estimated from $\mathbf{I}_{l o w}$.

Adaptive Merging Then a locally adaptive merging can be processed for each pixel according to the local mean. The $N$ compensated high-pass bands $L_{\frac{k i g h}{1}}^{*}, \ldots, L_{h i g h_{N}}^{*}$ are merged to one single compensated high-pass band $L_{h i g h_{\text {comp }}}^{*}$. Since only some of the MTF values have been measured for a limited set of bias and frequency 
values, missing MTF values are first linearly interpolated for the frequency range of the image sent to the printer $\left([0,150]\right.$ dpi in our experiments). Then for each pixel in $\mathbf{I}_{h i g h}$ the two bias levels $b_{n}$ and $b_{n+1}$ closest to the value $Y_{\text {low }}$ corresponding to that pixel are selected: $b_{n} \geq Y_{\text {low }} \geq b_{n+1}$. $L_{\text {high }}^{*}$ ism is linear interpolation between the compensated images $L_{\frac{\text { high }}{*}}^{*}$ at these two bias levels:

$$
L_{h i g h_{c o m p}}^{*}=w \cdot L_{\frac{\text { high }}{*}}^{*}+(1-w) \cdot L_{\overline{h i g h_{n+1}}}^{*}
$$

with:

$$
w=\frac{Y_{l o w}-b_{n+1}}{b_{n}-b_{n+1}}
$$

\section{DISCUSSION}

In this section we first compare the proposed algorithm to existing alternatives, then discuss possible evolutions.

Locally Adaptive Compensation Compared to Classic Deconvolution and Unsharp Masking Firstly, unlike the classic deconvolution and unsharp masking, the proposed locally adaptive compensation is based on image decomposition and thus only applied to a carefully restricted set of high-freqency content. This allows the results of the proposed algorithm to be free of haloing artifacts. Secondly, the proposed compensation is bias dependent, the MTF compensation is pixel-wise optimized to produce a resulting image with a constant $M T F=1$ across the printed image. To obtain similar results with unsharp masking, several filtering with decreasing size of blurring filter and different amount of unsharp masking would be needed. While theoretically possible, this solution is obviously not as elegant nor as precise as the proposed algorithm.

Orientation In this study, we have not considered the orientation of the high-frequency content as the measured MTF of our printing system is close to being orientation independent. In our implementation, the compensation is applied in the Fourier domain as a division by a $2 \mathrm{D}$ filter constructed by a $360^{\circ}$ polar rotation of the 1D MTF. It is thus easily expandable to a situation where the MTF depends on the rotation, by measuring the MTF at different orientations. The $2 \mathrm{D}$ filter will then be constructed by using the measured MTF at the corresponding angles and interpolation for intermediate angles.

Optimization It should be possible to reduce the number of bias levels used within the compensation by using a non-linear weights in Equation 12. These weights would be deduced from the bias dependency of the printer MTF. The approach of reducing the number of bias levels and therefore using a nonlinear interpolation would be interesting, since it would reduce the number of inverse Fourier transforms within our compensation algorithm.

It could even be profitable to use MTFs from bias levels which are not equally spaced. For example fewer bias levels in gray levels ranges where the bias dependency is weak and more in ranges where the bias dependency is strong. How to efficiently choose the number and values of the bias levels used for the compensation and how to interpolate between them is an optimization problem and would aim at providing full quality with as less computation power as possible.

\section{OVER-COMPENSATION}

In the previous section, the compensation of the MTF was based on objective measures by mean of a scanner. While according to the scanner-based measured objective results, the MTF compensation has achieved its goal, preliminary evaluation of the printed images by observers lead to interrogations about the amplitude of the perceived improvement. To further enhance the perceived quality of the reproduction, it might be necessary to over-compensate the MTF. In this scenario (see Figure 7), the MTF of the printing system is compensated for more than necessary and a stronger local contrast should be perceived in the resulting images. Such images could show better preserved local details and thus be judged as more accurate reproductions. 


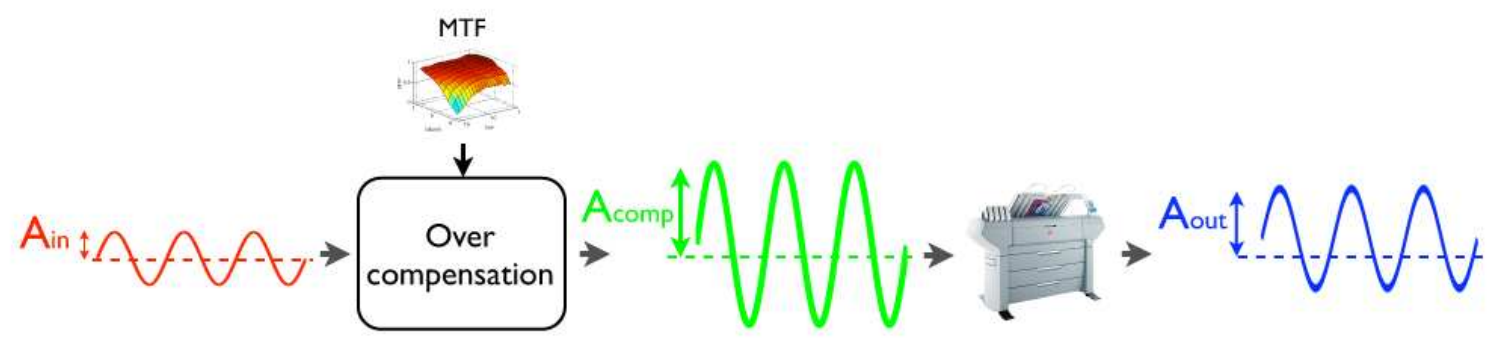

Figure 7. Principle of the MTF over-compensation: the Amplitude $A_{\text {in }}$ is over-compensated (over pre-amplified) by Overcompensation (Division by the $M T F$ in the Fourier domain) before printing, resulting in an output amplitude $A_{\text {out }}$ larger than the input $A_{i n}$.

How to Over-compensate? Unfortunately it is difficult to know how the over-compensation should be applied as it depends on the perception of the HVS. In our tests, we have thought of over-compensating as an underestimation of the printer MTF. Several operators can be applied to lower the MTF values, resulting in an over-compensation of the MTF:

- A global shift: $M T F_{\text {over }}=\max (M T F-\beta, 0)$ where $M T F_{\text {over }}$ is the modified MTF values and $\left.\left.\beta \in\right] 0,1\right]$ is the shifting coefficient. This shifting will have a stronger impact on high-frequency content.

- A global scaling factor: $M T F_{\text {over }}=\theta \times M T F$ where $M T F_{\text {over }}$ is the modified MTF values and $\left.\left.\theta \in\right] 0,1\right]$ is the scaling coefficient. Scaling will have a stronger impact on low-frequency content.

- A frequency dependent function, such as a power applied to the MTF values, might also have an interresting behavior.

Proposed Over-compensation Psychophysical experiments should be carried out to gain knowledge about the perception of the over-compensation. Preliminary tests show that a global scaling factor has a stronger perceived impact. An underestimation of $20 \%$ of the MTF, obtained by multiplying each MTF value by $\theta=0.8$ has been selected for its perceptually satisfying results.

\section{PSYCHOPHYSICAL EXPERIMENT}

The goal of this experiment was to evaluate the gain of the MTF compensation in spatial and color adaptive gamut mapping algorithms. ${ }^{1,22}$ To evaluate the compensation and over compensation of the printer MTF, three alternative degrees of compensation have been included in this study:

1. No compensation,

2. Locally adaptive MTF compensation,

3. Locally adaptive MTF over-compensation obtained by reducing the measured MTF by $20 \%$ by multiplying it by $\theta=0.8$, leading to a compensation by a factor $1 / \theta=1 / 0.8=1.25$, or $25 \%$.

Experiment Type and Observer Task In this category judgement experiment, the observers were presented 3 samples of a series of processed images along with a reference reproduction. They have to evaluate how close each reproduction is to the reference reproduction, on a scale of numbers from 1 to 7 where 1 represents the closest reproduction you can imagine and 7 represents the least accurate reproduction possible. A description of the accuracy for each level was proposed to help: 1) Most accurate you can imagine, 2) Highly accurate, 3) Very accurate, 4) Fairly accurate, 5) Moderately accurate, 6) Poorly accurate, 7) Least accurate reproduction possible. It was suggested to make the decision based on different parts of the image, to evaluate the fidelity of the reproduction of both colors and details, and look for possible artifacts. It is the accuracy of reproduction of the images which is evaluated, not the pleasantness. 
Images A total of 15 images were used in this experiment: 7 sRGB images from the ISO 12640-2:2004 standard $^{23}$ and 8 CIELAB/SCID images from the ISO 12640-3 $3^{24}$ (reproduced in Figure 8). Images were printed

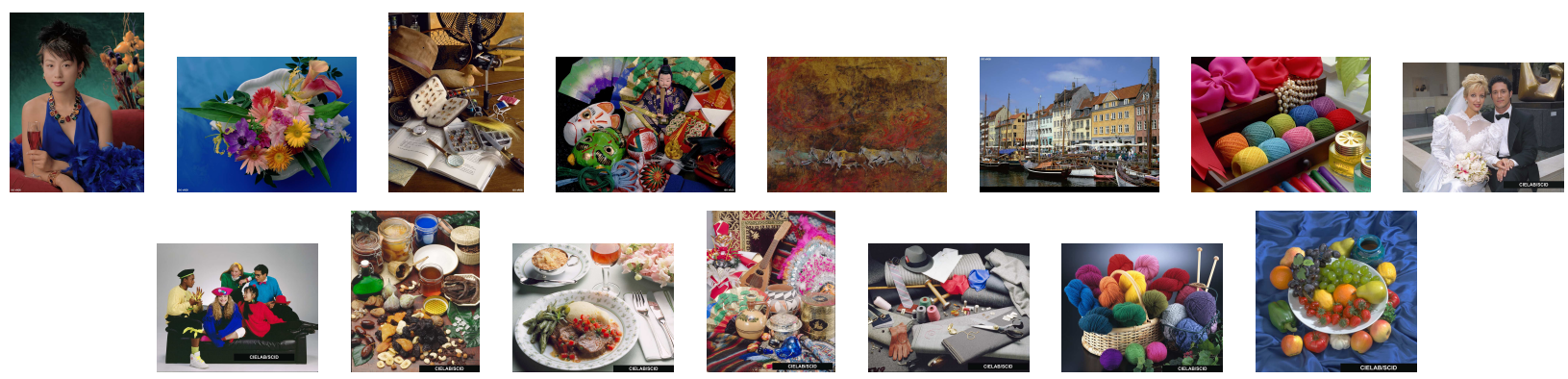

Figure 8. sRGB images from ISO 12640-2 (top) and CIELAB/SCID images from ISO 12640-3 (bottom).

at a resolution of 150 pixels per inch and a size of $1200 \times 1500$ pixels, 20.32 by $25.4 \mathrm{~cm}$ (i.e. 8 by 10 inches). The sRGB images were converted to CIELAB using the relative colorimetric intent of the sRGB profile. The CIELAB/SCID images are encoded in CIELAB colors. All the images were then gamut mapped in the CIELAB color space. ${ }^{1}$

Output device and medium The destination gamut was the gamut of an Océ ColorWave 600 printer using Océ Red Label paper and the printer highest quality setting Présentation mode. It was measured by a spectrophotometer GretagMacbeth Spectroscan using GretagMacBeth MeasureTool 5.0.8. The resulting images were then converted from CIELAB to the device CMYK using the relative colorimetric intent of a custom printer CMYK ICC version 2 profile. They were printed on a Océ ColorWave 600 printer using Océ Red Label paper with the color management disabled.

Observers and Viewing Conditions The test panel was constituted by 8 female and 9 male observers with normal vision and various degree of expertise in judging image quality. The 5 printed gamut-mapped candidates were presented in a controlled viewing room with a lighting system at a color temperature of 5200 Kelvins, a illuminance level of 450 lux \pm 75 and color rendering index of 96 . Our aim was to carry a thorough evaluation by having two sets of images with different input color gamuts and reference reproduction devices. The observers viewed simultaneously the reference image and the printed images from a distance of approximately $60 \mathrm{~cm}$. The experiment was then two-fold:

- For the sRGB image set, the observers were presented with a reference image on an EIZO ColorEdge CG221 display at a color temperature of 5200 Kelvins and luminance level of $120 \mathrm{~cd} / \mathrm{m}^{2}$. This set is rendered for sRGB display. Therefore a monitor capable of displaying the sRGB gamut is the most adapted reproduction device for this set of images.

- For the CIELAB/SCID image set, the observers were presented with a reference image printed on an Epson R800 printer using Epson Premium Glossy Photo Paper in the best printing quality mode. This set is rendered for the Perceptual Reference Medium Gamut (PRMG). The most appropriate way to reproduce these images is using a printing system able to reproduce the color gamut PRMG and with a good capability to reproduce details, such as the Epson R800 printer with Epson Premium Glossy Photo Paper.

Global Results Results cumulated over the 15 images and 17 observers in Figure 9 show that overall the over-compensation outperforms other alternatives. Each version is perceived as significantly different from the others, from the top rated: over-compensation, then compensation and no-compensation. Mean score for overcompensation and compensation are in category 3, mean score for no-compensation is in category 4. Overall, images with MTF compensation were evaluated as more accurate than without, and the over-compensated version was given the best scores. Printed over-compensated images appear to have a better preserved local 


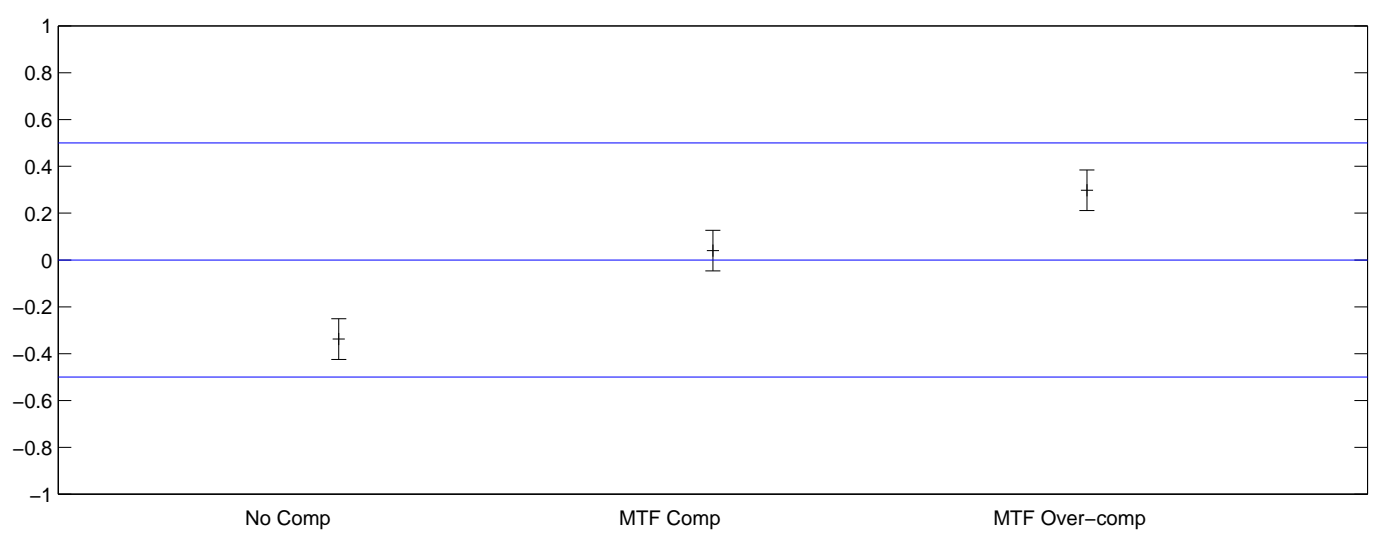

Figure 9. Z-scores resulting of our experiment (category scaling), average over 15 images and 17 observers.

contrast when compared to non-compensated and compensated reproductions of the same images. This is a clear indication that MTF compensation fulfills its goal of producing more accurate reproductions. Further study will be necessary to find an optimal over-compensation operator.

\section{CONCLUSIONS}

In this paper we have proposed an approach to compensate for the printer modulation transfer function. A new compensation algorithm has been presented. It fully exploits both the frequency and the bias dependency of the measured MTF values. The category judgement experiment on photographic images has delivered encouraging results: MTF compensation fulfills its goal of producing more accurate reproductions. More studies remain necessary to fully apprehend the benefits and drawbacks of the MTF compensation and to find optimal parameters for over-compensation, including in the case of noisy images. In a printing workflow composed of an input device (such as a scanner/ digital camera or a monitor) and an output system (such as a printing system or a monitor), the MTF compensation should consider the MTFs of both devices and process the captured image once to compensate for the two MTFs. The use of MTF compensation in other imaging workflows should also be investigated.

\section{ACKNOWLEDGMENTS}

We would like to thank Professor Isabelle Bloch for her thoughtful comments.

\section{REFERENCES}

[1] Bonnier, N., [Contributions to Spatial Gamut Mapping Algorithms], Telecom ParisTech, PhD Thesis, Paris, France (2008).

[2] Jang, W. and Allebach, J. P., "Characterization of printer MTF," in [Proceedings of the ISETTSPIE Electronic Imaging Conference, Image Quality and System Performance III], Cui, L. C. and Miyake, Y., eds., 6059, 1-12, SPIE (2006).

[3] Lindner, A. J., Bonnier, N., Schmitt, F., and Leynadier, C., "Evaluation of characterization methods of printer MTF," in [Proceedings of the ISET/SPIE Electronic Imaging Conference, Image Quality and System Performance V], Farnand, S. P. and Gaykema, F., eds., 6808, 6808061-68080612, SPIE, San Jose, California (2008).

[4] McCann, J. J., "Color gamut mapping using spatial comparisons," in [Proceedings of the ISETTSPIE Electronic Imaging Conference, Color Imaging: Device-Independent Color, Color Hardcopy, and Graphic Arts $V I]$, Eschbach, R. and Marcu, G. G., eds., 4300, 126-130, SPIE (2000).

[5] Sun, P.-L., [The Influence of Image Characteristics on Colour Gamut Mapping], University of Derby PhD Thesis, Derby, UK (2002). 
[6] Shannon, C. E., "Communication in the presence of noise," Proc. Institute of Radio Engineers 37, 10-21 (1949).

[7] ISO 12233, [ISO 12233:2000 Photography - Electronic still picture cameras - Resolution measurements], ISO (2000).

[8] ISO 16067-1, [ISO 16067-1:2003 Photography - Spatial resolution measurements of electronic scanners for photographic images - Part 1: Scanners for reflective media], ISO (2003).

[9] Arney, J., Anderson, P., Mehta, P., and Ayer, K., "The MTF of a printing systems," in [Proceedings of the ISET/SPIE NIP16: International Conference on Digital Printing Technologies], 367-369 (2000).

[10] Hasegawa, J., Hwang, T.-Y., Kim, H.-C., Kim, D.-W., and Choi, M.-H., "Measurement-based objective metric for printer resolution," in [Proceedings of the ISET/SPIE Electronic Imaging Conference, Image Quality and System Performance IV], Cui, L. C. and Miyake, Y., eds., 6494(1), 64940D, SPIE (2007).

[11] Bang, Y., Kim, S. H., and Choi, D. C., "Printer resolution measurement based on slanted edge method," in [Proceedings of the ISET/SPIE Electronic Imaging Conference, Image Quality and System Performance $V]$, Farnand, S. P. and Gaykema, F., eds., 680807-680815, SPIE (2008).

[12] Lindner, A. J., Bonnier, N., Schmitt, F., and Leynadier, C., "Measurement of printer MTFs," in [Proceedings of the ISET/SPIE Electronic Imaging Conference, Image Quality and System Performance VI], Farnand, S. P. and Gaykema, F., eds., SPIE, San Jose, California (2009).

[13] Wyszecki and Styles, [Color Science, Concepts and Methods, Quantitative Data and Formulae, Second Edition], Wiley Interscience, Derby, UK (2000).

[14] Keelan, B., [Handbook of Image Quality: Characterization and Prediction], Marcel Dekker Inc. (2002).

[15] Wiener, N., [Extrapolation, Interpolation, and Smoothing of Stationary Time Series], Wiley (1949).

[16] Chan, T. F. and Shen, J., "Theory and computation of variational image deblurring," tech. rep., UCLA and University of Minnesota (2007).

[17] Russ, J. C., [The Image Processing Handbook], CRC Press (2007).

[18] Lindner, A. J., Master Thesis Report: Measurement and Compensation of Printer Modulation Transfer Functions, Master's thesis, Telecom Paristech (2007).

[19] Bonnier, N., Schmitt, F., Hull, M., and Leynadier, C., "Spatial and color adaptive gamut mapping: A mathematical framework and two new algorithms," in [Proceedings of the 15th ISET/SID Color Imaging Conference], 267-272 (2007).

[20] Tomasi, C. and Manduchi, R., "Bilateral filtering for gray and color images," in [Proceedings of the Sixth International Conference on Computer Vision], 839-846, IEEE Computer Society, Washington, DC, USA (1998).

[21] Bonnier, N., Schmitt, F., and Leynadier, C., "Improvements in spatial and color adaptive gamut mapping algorithms," in [Proceedings of ISGT/SPIE 4th European Conference on Colour in Graphics, Imaging and Vision], 341-346 (2008).

[22] Bonnier, N., Schmitt, F., and Leynadier, C., "Compensating the printer's modulation transfer function in spatial and color adaptive rendering workflows," in [Proceedings of the 16th ISET/SID Color Imaging Conference], (2008).

[23] ISO 12640-2, [ISO 12640-2:2004 Graphic technology - Prepress digital data exchange - Part 2: XYZ/sRGB encoded standard colour image data (XYZ/SCID)], ISO (2004).

[24] ISO 12640-3, [ISO 12640-3:2007 Graphic technology - Prepress digital data exchange - Part 3: CIELAB standard colour image data (CIELAB/SCID)], ISO (2007). 